\title{
First Order Reactant in Magneto-Hydrodynamic Turbulence before the Final Period of Decay in Presence of Dust Particle
}

\author{
M. A. K. Azad, M. A. Aziz and M. S. Alam Sarker \\ Department of Applied Mathematics University of Rajshahi-6205, Bangladesh
}

\begin{abstract}
Following Deissler's theory, the decay for the concentration fluctuation of a dilute contaminant undergoing a first order chemical reaction in MHD turbulence at times before the final period in presence of dust particle is studied and have considered correlations between fluctuating quantities at two and three point. Two and three point correlation equations are obtained and the set of equations is made to determinate by neglecting the quadruple correlations in comparison to the second and third order correlations. The correlation equations are converted to spectral form by taking their Fourier transforms. Finally we obtained the Decay law of magnetic energy for the concentration fluctuations before the final period in presence of dust particle by integrating the energy spectrum over all wave numbers.
\end{abstract}

Keywords: MHD Turbulence, First order reactant, Dust particle, Decay before the final period .

\section{Introduction}

A dust particle in air, or in any other gas, has a much larger inertia than the equivalent volume of air and will not therefore participate readily in turbulent fluctuations. The relative motion of dust participate and the air will dissipate energy because of the drag between dust and air, and extract energy from turbulent intensity is reduced than the Reynolds stresses will be decreased and the force required to maintain a given flow rate will likewise be reduced. (Sarker,1993) discussed the vorticity covariance of dusty fluid turbulence in a rotating frame.

(Deissler,1958) developed a theory "decay of homogeneous turbulence for times before the final period". Using Deissler's theory, (Loefler and Deissler,1961) studied the decay of temperature fluctuations in homogeneous turbulence before the final period. In their approach they considered the two and three-point correlation equations and solved these equations after neglecting fourth and higher order correlation terms. Using Deissler theory, (Kumar and Patel,1974) studied the first-order reactant in homogeneous turbulence before the final period of decay for the case of multi-point and single-time correlation. (Kumar and Patel,1975) extended their problem, (Kumar and Patel,1974) for the case of multi-point and multi-time concentration correlation. (Patel, 1976) also studied in detail the same problem to carry out the numerical results. (Sarker and Kishore,1991) studied the decay of MHD turbulence at time before the final period using (Chandrasakher's relation,1951). (Sarker and Islam,2001) studied the decay of
MHD turbulence before the final period for the case of multipoint and multi-time. (Azad and Sarker,2003) studied the Decay of MHD turbulence before the final period for the case of multi-point and multi-time in presence of dust particle. (Islam and Sarker,2001) studied the first order reactant in MHD turbulence before the final period of decay for the case of multi-point and multi-time. (Sarker and Islam,2001) also studied the first order reactant in MHD turbulence before the final period of decay.

In our present work, we studied the magnetic field fluctuation of concentration of a dilute contaminant undergoing a first order chemical reaction in dusty fluid MHD turbulence before the final period of decay. Here, we have considered the two-point and three-point correlation equations and solved these equations after neglecting the fourth-order correlation terms. Finally we obtained the decay law for magnetic field energy fluctuation of concentration of dilute contaminant undergoing a first order chemical reaction in dusty fluid MHD turbulence comes out to be

$$
\left\langle h^{2}\right\rangle=\exp \left[-R\left(t-t_{0}\right)\left[A\left(t-t_{0}\right)^{-3 / 2}+\exp [f s] B\left(t-t_{0}\right)^{-5}\right]\right.
$$

where $\left\langle h^{2}\right\rangle$ denotes the total energy (mean square of the magnetic field fluctuations of concentration), $t$ is the time and $\mathrm{A}, \mathrm{B}$ and $\mathrm{t}_{0}$ are constants. $\mathrm{s}$ is an another arbitrary constant which is defined by the equation (36).

\footnotetext{
* Corresponding author: E-mail: akmrashidulalam@yahoo.com
} 


\section{Basic equations}

The equation of motion and continuity for viscous, incompressible MHD dusty fluid turbulent flow are given by (Chandrasekhar,1951) as

$\frac{\partial u_{i}}{\partial t}+\frac{\partial}{\partial x_{k}}\left(u_{i} u_{k}-h_{i} h_{k}\right)=-\frac{\partial w}{\partial x_{i}}+v \frac{\partial^{2} u_{i}}{\partial x_{k} \partial x_{k}}+\left(u_{i}-v_{i}\right) f$

$\frac{\partial h_{i}}{\partial t}+\frac{\partial}{\partial x_{k}}\left(h_{i} u_{k}-u_{i} h_{k}\right)=\lambda \frac{\partial^{2} h_{i}}{\partial x_{k} \partial x_{k}}$

$\frac{\partial v_{i}}{\partial t}+v_{k} \frac{\partial v_{i}}{\partial x_{k}}=-\frac{k}{m_{s}}\left(v_{i}-u_{i}\right)$

with

$\frac{\partial u_{i}}{\partial x_{i}}=\frac{\partial v_{i}}{\partial x_{i}}=\frac{\partial h_{i}}{\partial x_{i}}=0$

The subscripts can take on the values 1,2 or 3 .

Here, $\mathrm{u}_{\mathrm{i}}$, turbulent velocity component; $\mathrm{h}_{\mathrm{i}}$, magnetic field fluctuation component, $\mathrm{v}_{\mathrm{i}}$, dust velocity component

$W(\hat{x}, t)=\frac{p}{\rho}+\frac{1}{2}\left\langle h^{2}\right\rangle$,

total MHD pressure inclusive of potential force;

$p(\hat{x}, t)=$ hydrodynamic pressure,

$\rho=$ fluid density,

$p_{M}=\frac{v}{\lambda}$, magnetic prandtl number,

$p_{r}=\frac{v}{\gamma}$, prandtl number,

$v$ = kinematic viscosity,

$\gamma=\frac{\mathrm{K}}{\rho c_{p}}$, thermal diffusivity,

$\lambda=(4 \pi \mu \sigma)^{-1}$, magnetic diffusivity,

$\mathrm{c}_{\mathrm{p}}=$ heat capacity at constant pressure,

$\in_{m k i}=$ alternating tensor,

$f=\frac{k N}{\rho}$, dimension of frequency; $\mathrm{N}$,

constant number density of dust particle,

$m_{s}=\frac{4}{3} \pi R^{3}{ }_{s} \rho_{s}$, mass of single spherical dust particle of radius $R_{s}$,

$\rho_{s}=$ constant density of the material in dust particle,

$\mathrm{x}_{\mathrm{k}}=$ Space co-ordinate, the subscripts can take on the values 1,2 or 3 .

\section{Two-point correlation and spectral equations}

Under the conditions that (i) the turbulence and the concentration magnetic field are homogeneous (ii) the chemical reaction has no effect on the velocity field and (iii) the reaction rate and the magnetic diffusivity are constant, the induction equation of a magnetic field fluctuation of concentration of a dilute contaminant undergoing a first order chemical reaction at the points $\mathrm{p}$ and $\mathrm{p}$ Separated by the vector $\hat{r}$ could be written as

$$
\begin{array}{r}
\frac{\partial h_{i}}{\partial t}+u_{k} \frac{\partial h_{i}}{\partial x_{k}}-h_{k} \frac{\partial u_{i}}{\partial x_{k}}=\lambda \frac{\partial^{2} h_{i}}{\partial x_{k} \partial x_{k}}-R h_{i} \\
\text { and } \frac{\partial h_{i}}{\partial t}+u_{k} \frac{\partial h_{i}}{\partial x_{k}}-h_{k} \frac{\partial u_{i}}{\partial x_{k}}=\lambda \frac{\partial^{2} h_{i}}{\partial x_{k} \partial x_{k}}-R h_{i}
\end{array}
$$

Where $\mathrm{R}$ is the constant reaction rate.

Multiplying equation (5) by $h_{j}$ and (6) by $h_{i}$, adding and taking ensemble average, we get

$$
\begin{array}{r}
\frac{\partial\left\langle h_{i} h_{j}^{\prime}\right\rangle}{\partial t}+\frac{\partial}{\partial x}\left[\left\langle u_{k} h_{i} h_{j}^{\prime}\right\rangle-\left\langle h_{k} u_{i} h_{j}^{\prime}\right\rangle\right]+\frac{\partial}{\partial x_{k}^{\prime}}\left[\left\langle u_{k}^{\prime} h_{i} h_{j}^{\prime}\right\rangle-\left\langle h_{k}^{\prime} u_{j}^{\prime} h_{i}\right\rangle\right] \\
=\lambda\left[\frac{\partial^{2}\left\langle h_{i} h_{j}^{\prime}\right\rangle}{\partial x_{k} \partial x_{k}}+\frac{\partial^{2}\left\langle h_{i} h_{j}^{\prime}\right\rangle}{\partial x_{k}^{\prime} \partial x_{k}^{\prime}}\right]-2 R\left\langle h_{i} h_{j}^{\prime}\right\rangle \quad(7)
\end{array}
$$

Angular bracket $\langle\ldots . . . . . . . . . .$.$\rangle is used to denote an ensemble$ average

Using the transformations,

$\frac{\partial}{\partial r_{k}}=-\frac{\partial}{\partial x_{k}}=\frac{\partial}{\partial x_{k}^{\prime}}$

and the Chandrasekhar relations [8]

$\left\langle u_{k} h_{i} h_{j}^{\prime}\right\rangle=-\left\langle u_{k}^{\prime} h_{i} h_{j}^{\prime}\right\rangle,\left\langle u_{j}^{\prime} h_{i} h_{k}^{\prime}\right\rangle=-\left\langle u_{i} h_{k} h_{j}^{\prime}\right\rangle$

Equation (7) becomes

$$
\begin{aligned}
& {\left[\frac{\partial\left\langle h_{i} h_{j}^{\prime}\right\rangle}{\partial t}+2 \frac{\partial}{\partial r_{k}}\left[\left\langle u_{k}^{\prime} h_{i} h_{j}^{\prime}\right\rangle-\left\langle u_{i} h_{k} h_{j}^{\prime}\right\rangle\right]\right]} \\
& =2 \lambda \frac{\partial^{2}\left\langle h_{i} h_{j}^{\prime}\right\rangle}{\partial r_{k} \partial r_{k}}-2 R\left\langle h_{i} h_{j}^{\prime}\right\rangle
\end{aligned}
$$

Now we write equation (10) in spectral form in order to reduce it to an ordinary differential equation by use of the following three-dimensional Fourier transforms.

$$
\left.\left\langle h_{i} h_{j}^{\prime}(\hat{r})\right\rangle=\int_{-\infty}^{\infty}\left\langle\psi_{i} \psi_{j}^{\prime}(\hat{k})\right\rangle \exp \hat{\mathbf{E}}(\hat{k} . \hat{r})\right] d \hat{k}
$$


$\left\langle u_{i} h_{k} h_{j}^{\prime}(\hat{r})\right\rangle=\int^{\infty}\left\langle\alpha_{i} \psi_{k} \psi_{j}^{\prime}(\hat{k})\right\rangle \exp [\hat{\mathbf{E}}(\hat{k} . \hat{r})] d \hat{K}$

Interchanging $\mathrm{i}^{-\infty}$ and $\mathrm{j}$, points $\mathrm{p}$ and then,

$$
\begin{aligned}
& \left\langle u_{k}^{\prime} h_{i} h_{j}^{\prime}(\hat{r})\right\rangle=\left\langle u_{k} h_{i} h_{j}^{\prime}(-\hat{r})\right\rangle=\int_{-\infty}^{\infty}\left\langle\alpha_{k} \psi_{i} \psi_{j}^{\prime}(-\hat{k})\right\rangle \\
& \exp [\hat{\mathbf{i}}(\hat{k} . \hat{r})] \hat{k}
\end{aligned}
$$

Substituting of equation (11) to (13) in to equation (10) leads to the Spectral equation

$$
\begin{aligned}
& \frac{\partial\left\langle\psi_{i} \psi_{j}^{\prime}(\hat{k})\right\rangle}{\partial t}+2 \lambda K^{2}\left\langle\psi_{i} \psi_{j}^{\prime}(\hat{k})\right\rangle+2 R\left\langle\psi_{i} \psi_{j}^{\prime}(\hat{k})\right\rangle \\
& =2 i k\left[\left\langle\alpha_{i} \psi_{i} \psi_{j}^{\prime}(\hat{k})\right\rangle-\left\langle\alpha_{k} \psi_{i} \psi_{j}^{\prime}(-\hat{k})\right\rangle\right] \\
& \Rightarrow \frac{\partial\left\langle\psi_{i} \psi_{j}^{\prime}(\hat{k})\right\rangle}{\partial t}+2 \lambda\left[K^{2}+\frac{R}{\lambda}\right]\left\langle\psi_{i} \psi_{j}^{\prime}(\hat{k})\right\rangle \\
& =2 i k\left[\left\langle\alpha_{i} \psi_{i} \psi_{j}^{\prime}(\hat{k})\right\rangle-\left\langle\alpha_{k} \psi_{i} \psi_{j}^{\prime}(-\hat{k})\right\rangle\right]
\end{aligned}
$$

The tensor equation (15) becomes a scalar equation by contraction of the indices $i$ and $j$

$$
\begin{aligned}
& \frac{\partial\left\langle\psi_{i} \psi_{i}^{\prime}(\hat{k})\right\rangle}{\partial t}+2 \lambda\left[K^{2}+\frac{R}{\lambda}\right]\left\langle\psi_{i} \psi_{i}^{\prime}(\hat{k})\right\rangle \\
& =2 i k\left[\left\langle\alpha_{i} \psi_{i} \psi_{i}^{\prime}(\hat{k})\right\rangle-\left\langle\alpha_{k} \psi_{i} \psi_{i}^{\prime}(-\hat{k})\right\rangle\right]
\end{aligned}
$$

The term on the right hand side of equation (16) is called energy transfer term while the second term on the left hand side is the dissipation term.

\section{Three-point correlation and spectral equations:}

Similar Procedure can be used to find the three points correlation equation. For this purpose we take the momentum equation of dusty fluid MHD turbulence at the point $\mathrm{P}$ and the induction equations of magnetic field fluctuation, governing the concentration of a dilute contaminant undergoing a first order chemical reaction at $P$ Cand $P$ @Oseparated by the vectors $\hat{r}$ and $\hat{r}^{\prime}$ as

$\frac{\partial u_{l}}{\partial t}+u_{k} \frac{\partial u_{l}}{\partial k_{k}}-h_{k} \frac{\partial h_{l}}{\partial x_{k}}=-\frac{\partial w}{\partial x_{l}}+v \frac{\partial^{2} u_{l}}{\partial x_{k} \partial x_{k}}+f\left(u_{l}-v_{l}\right)$

$\frac{\partial h_{i}^{\prime}}{\partial t}+u_{k}^{\prime} \frac{\partial h_{i}^{\prime}}{\partial x_{k}^{\prime}}-h_{k}^{\prime} \frac{\partial u_{i}^{\prime}}{\partial x_{k}^{\prime}}=\lambda \frac{\partial^{2} h_{i}^{\prime}}{\partial x_{k}^{\prime} \partial x_{k}^{\prime}}-R h_{i}^{\prime}$

$\frac{\partial h_{j}^{\prime \prime}}{\partial t}+u_{k}^{\prime \prime} \frac{\partial h_{j}^{\prime \prime}}{\partial x_{k}^{\prime \prime}}-h_{k}^{\prime \prime} \frac{\partial u_{j}^{\prime \prime}}{\partial x_{k}^{\prime \prime}}=\lambda \frac{\partial^{2} h_{j}^{\prime \prime}}{\partial x_{k}^{\prime \prime} \partial x_{k}^{\prime \prime}}-R h_{j}^{\prime \prime}$ where $W(\hat{x}, t)=\frac{P}{\rho}+\frac{1}{2}\left\langle h^{2}\right\rangle$, total MHD pressure inclu sive of potential force $P(\hat{x}, t)$, hydrodynamic pressure; $\in_{m k i}$, alternating tensor, $f=\frac{k N}{\rho}$ dimension frequency; $\mathrm{N}$, constant number density of dust particle.

Multiplying equation (17) by $h_{i}^{\prime} h_{j}^{\prime \prime}$ (18) by $u_{l} h_{j}^{\prime \prime}$ and (19) by $u_{l} h_{i}^{\prime}$, adding and taking ensemble average, we obtain

$$
\begin{gathered}
\frac{\partial\left\langle u_{l} h_{i}^{\prime} h_{j}^{\prime \prime}\right\rangle}{\partial t}+\frac{\partial}{\partial x_{k}}\left[\left\langle u_{k} u_{l} h_{i}^{\prime} h_{j}^{\prime \prime}\right\rangle-\left\langle h_{k} h_{l} h_{i}^{\prime} h_{j}^{\prime \prime}\right\rangle\right]+\frac{\partial}{\partial x_{k}^{\prime}} . \\
{\left[\left\langle u_{l} u_{k}^{\prime} h_{i}^{\prime} h_{j}^{\prime \prime}\right\rangle-\left\langle u_{l} u_{i}^{\prime} h_{k}^{\prime} h_{j}^{\prime \prime}\right\rangle\right]} \\
+\frac{\partial}{\partial x_{k}^{\prime \prime}}\left[\left\langle u_{l} u_{k}^{\prime \prime} h_{i}^{\prime} h_{j}^{\prime \prime}\right\rangle-\left\langle u_{l} u_{j}^{\prime \prime} h_{i}^{\prime} h_{j}^{\prime \prime}\right\rangle\right] \\
=-\frac{\partial\left\langle w h_{i}^{\prime} h_{j}^{\prime \prime}\right\rangle}{\partial x_{l}}+v \frac{\partial^{2}\left\langle u_{l} h_{i}^{\prime} h_{j}^{\prime \prime}\right\rangle}{\partial x_{k} \partial x_{k}}+\lambda \\
{\left[\frac{\partial^{2}\left\langle u_{l} h_{i}^{\prime} h_{j}^{\prime \prime}\right\rangle}{\partial x_{k}^{\prime} \partial x_{k}^{\prime}}+\frac{\partial^{2}\left\langle u_{l} h_{i}^{\prime} h_{j}^{\prime \prime}\right\rangle}{\partial x_{k}^{\prime \prime} \partial x_{k}^{\prime \prime}}\right]} \\
-2 R\left\langle u_{l} h_{i}^{\prime} h_{j}^{\prime \prime}\right\rangle+f\left(\left\langle u_{l} h_{i}^{\prime} h_{j}^{\prime \prime}\right\rangle-\left\langle v_{l} h_{i}^{\prime} h_{j}^{\prime \prime}\right)\right.
\end{gathered}
$$

Using the transformations

$\frac{\partial}{\partial x_{k}}=-\left(\frac{\partial}{\partial r_{k}}+\frac{\partial}{\partial r_{k}^{\prime}}\right), \frac{\partial}{\partial x_{k}^{\prime}}=\frac{\partial}{\partial r_{k}}, \quad \frac{\partial}{\partial x_{k}^{\prime \prime}}=\frac{\partial}{\partial r_{k}^{\prime}}$

into equation (20)

$\frac{\partial\left\langle u_{l} h_{i}^{\prime} h_{j}^{\prime \prime}\right\rangle}{\partial t}-\lambda\left[\left(1+P_{M}\right) \frac{\partial^{2}\left\langle u_{l} h_{i}^{\prime} h_{j}^{\prime \prime}\right\rangle}{\partial r_{k} \partial r_{k}}+\left(1+P_{M}\right)\right.$

$\left.\frac{\partial^{2}\left\langle u_{l} h_{i}^{\prime} h_{j}^{\prime \prime}\right\rangle}{\partial r_{k}^{\prime} \partial r_{k}^{\prime}}+2 P_{M} \frac{\partial^{2}\left\langle u_{l} h_{i}^{\prime} h_{j}^{\prime \prime}\right\rangle}{\partial r_{k} \partial r_{k}}\right]$

$=\frac{\partial}{\partial r_{k}}\left\langle u_{l} u_{k} h_{i}^{\prime} h_{j}^{\prime \prime}\right\rangle+\frac{\partial}{\partial r_{k}^{\prime}}\left\langle u_{l} u_{k} h_{i}^{\prime} h_{j}^{\prime \prime}\right\rangle$

$-\frac{\partial}{\partial r_{k}^{\prime}}\left\langle h_{l} h_{k} h_{i}^{\prime} h_{j}^{\prime \prime}\right\rangle-\frac{\partial}{\partial r_{k}^{\prime}}\left\langle h_{l} h_{k} h_{i}^{\prime} h_{j}^{\prime \prime}\right\rangle$ 
$-\frac{\partial}{\partial r_{k}}\left\langle u_{l} u_{k}^{\prime} h_{i}^{\prime} h_{j}^{\prime \prime}\right\rangle+\frac{\partial}{\partial r_{k}}\left\langle u_{l} u_{i}^{\prime} h_{k}^{\prime} h_{j}^{\prime \prime}\right\rangle-\frac{\partial}{\partial r_{k}^{\prime}}$

$\left\langle u_{l} u_{k}^{\prime \prime} h_{i}^{\prime} h_{j}^{\prime \prime}\right\rangle+\frac{\partial}{\partial r_{k}^{\prime}}\left\langle u_{l} u_{j}^{\prime \prime} h_{i}^{\prime} h_{j}^{\prime \prime}\right\rangle+\frac{\partial}{\partial r_{l}}\left\langle w h_{i}^{\prime} h_{j}^{\prime \prime}\right\rangle+\frac{\partial}{\partial r_{l}^{\prime}}\left\langle w h_{i}^{\prime} h_{j}^{\prime \prime}\right\rangle \cdot$

$-2 R\left\langle u h_{i}^{\prime} h_{j}^{\prime \prime}\right\rangle+f\left[\left\langle u_{l} h_{i}^{\prime} h_{j}^{\prime \prime}\right\rangle-\left\langle v_{l} h_{i}^{\prime} h_{j}^{\prime \prime}\right\rangle\right]$

In order to write the equation (22) to spectral form, we can define the following six dimensional Fourier transforms:

$\left\langle u_{l} h_{i}^{\prime}(\hat{r}) h_{j}^{\prime \prime}\left(\hat{r}^{\prime}\right)\right\rangle=\int^{\infty} \int^{\infty}\left\langle\phi_{l} \beta_{i}^{\prime}(\hat{k}) \beta_{j}^{\prime \prime}(\hat{k})\right\rangle$

$\exp \left[\hat{i}\left(\hat{k} \cdot \hat{r}+\hat{k}^{\prime} \cdot \hat{r}^{\prime}\right)\right] \hat{k} d \hat{k}^{\prime}$

$\left\langle u_{l} u_{k}^{\prime} h_{i}^{\prime}(\hat{r}) h_{j}^{\prime \prime}\left(\hat{r}^{\prime}\right)\right\rangle=\int_{-\infty}^{\infty} \int_{-\infty}^{\infty}\left\langle\phi_{l} \phi_{k}^{\prime}(\hat{k}) \beta_{i}^{\prime}(\hat{k}) \beta_{j}^{\prime \prime}\left(\hat{k}^{\prime}\right)\right\rangle$

$\left.\exp \hat{\mathbf{i}}\left(\hat{k} \cdot \hat{r}+\hat{k}^{\prime} \cdot \hat{r}^{\prime}\right)\right] \hat{k} d \hat{k}^{\prime}$

$\left\langle u_{l} u_{i}^{\prime}(\hat{r}) h_{k}^{\prime}(\hat{r}) h_{j}^{\prime \prime}\left(\hat{r}^{\prime}\right)\right\rangle=\int_{-\infty}^{\infty} \int_{-\infty}^{\infty}\left\langle\phi_{l} \phi_{i}^{\prime}(\hat{k}) \beta_{k}^{\prime}(\hat{k}) \beta_{j}^{\prime \prime}\left(\hat{k}^{\prime}\right)\right\rangle$

$\left.\exp \left[\hat{k} \cdot \hat{k}+\hat{k}^{\prime} \cdot \hat{r}^{\prime}\right)\right] \hat{k} d \hat{k}^{\prime}$

$\left\langle u_{l} u_{k} h_{i}^{\prime}(\hat{r}) h_{j}^{\prime \prime}\left(\hat{r}^{\prime}\right)\right\rangle=\int_{-\infty-\infty}^{\infty} \int_{\hat{n}^{\prime}}^{\infty}\left\langle\phi_{l} \phi_{k} \beta_{i}^{\prime \prime}(\hat{k}) \beta_{j}^{\prime \prime}\left(\hat{k}^{\prime}\right)\right\rangle_{1}$

$\exp \left[\hat{\mathbf{E}}\left(\hat{k} \cdot \hat{r}+\hat{k}^{\prime} \cdot \hat{r}^{\prime}\right)\right] d \hat{k} d \hat{k}^{\prime}$

$\left\langle h_{l} h_{k} h_{i}^{\prime}(\hat{r}) h_{j}^{\prime \prime}\left(\hat{r}^{\prime}\right)\right\rangle=\int_{-\infty}^{\infty} \int_{-\infty}^{\infty}\left\langle\beta_{l} \beta_{k} \beta_{i}^{\prime}(\hat{k}) \beta_{j}^{\prime \prime}\left(\hat{k}^{\prime}\right)\right\rangle_{\prime}$

$\exp \left[\hat{\mathbf{E}}\left(\hat{k} \cdot \hat{r}+\hat{k}^{\prime} \cdot \hat{r}^{\prime}\right)\right] d \hat{k} d \hat{k}^{\prime}$

$\left\langle w h_{i}^{\prime}(\hat{r}) h_{j}^{\prime \prime}\left(\hat{r}^{\prime}\right)\right\rangle=\int_{-\infty-\infty}^{\infty} \int_{i}^{\infty}\left\langle\gamma \beta_{i}^{\prime}(\hat{k}) \beta_{j}^{\prime \prime}\left(\hat{k}^{\prime}\right)\right\rangle$

$\exp \left[\hat{\mathrm{i}}\left(\hat{k} \cdot \hat{r}+\hat{k}^{\prime} \cdot \hat{r}^{\prime}\right)\right] \hat{k} d \hat{k}^{\prime}$

$\left\langle v_{l} h_{i}^{\prime}(\hat{r}) h_{j}^{\prime \prime}\left(\hat{r}^{\prime}\right)\right\rangle=\int_{-\infty-\infty}^{\infty} \int_{l}^{\infty}\left\langle\delta_{l} \beta_{i}^{\prime}(\hat{k}) \beta_{j}^{\prime \prime}\left(\hat{k}^{\prime}\right)\right\rangle$

$\exp \left[\hat{i}\left(\hat{k} \cdot \hat{r}+\hat{k}^{\prime} \cdot \hat{r}^{\prime}\right)\right] \hat{k} d \hat{k}^{\prime}$

Interchanging the points $P^{\prime}$ and $P$ ” along with the indices $i$ and $j$, result in the relations

$\left\langle u_{l} u_{k}^{\prime \prime} h_{j}^{\prime \prime} h_{i}^{\prime}\right\rangle=\left\langle u_{l} u_{k}^{\prime} h_{i}^{\prime} h_{j}^{\prime \prime}\right\rangle,\left\langle u_{l} u_{j}^{\prime \prime} h_{i}^{\prime} h_{j}^{\prime \prime}\right\rangle$

$=\left\langle u_{l} u_{i}^{\prime} h_{k}^{\prime} h_{j}^{\prime \prime}\right\rangle, \quad\left\langle u_{l} u_{j}^{\prime \prime} h_{i}^{\prime}\right\rangle=\left\langle u_{l} u_{i}^{\prime} h_{j}^{\prime \prime}\right\rangle$
By use of this facts(30) and equations (23)-(29), the equation (22) may be transformed as

$$
\begin{aligned}
& \frac{\partial\left\langle\phi_{l} \beta_{i}^{\prime} \beta_{j}^{\prime \prime}\right\rangle}{\partial t}+\lambda\left[\left(1+P_{M}\right) k^{2}+\left(1+P_{M}\right) k^{\prime 2}\right. \\
& \left.+2 P_{M} k_{k} k_{k}^{\prime}+2 \frac{R}{\lambda}-\frac{f}{\lambda}\right]\left\langle\phi_{l} \beta_{i}^{\prime} \beta_{j}^{\prime \prime}\right\rangle \\
& =i\left(k_{k}+k_{k}^{\prime}\right)\left\langle\phi_{l} \phi_{k} \beta_{i}^{\prime} \beta_{j}^{\prime \prime}\right\rangle-i\left(k_{k}+k_{k}^{\prime}\right)\left\langle\beta_{l} \beta_{k} \beta_{i}^{\prime} \beta_{j}^{\prime \prime}\right\rangle \\
& -i\left(k_{k}+k_{k}^{\prime}\right)\left\langle\phi_{l} \phi_{k}^{\prime} \beta_{i}^{\prime} \beta_{j}^{\prime \prime}\right\rangle+i\left(k_{k}+k^{\prime}\right)\left\langle\phi_{l} \phi_{i}^{\prime} \beta_{i}^{\prime} \beta_{j}^{\prime \prime}\right\rangle \\
& +i\left(k_{l}+k_{l}^{\prime}\right)\left\langle\gamma \beta_{i}^{\prime} \beta_{j}^{\prime \prime}\right\rangle-f\left\langle\delta_{l} \beta_{i}^{\prime} \beta_{j}^{\prime \prime}\right\rangle
\end{aligned}
$$

The tensor equation (31) can be converted to scalar equation by contraction of the indices $i$ and $j$

$$
\begin{aligned}
& \frac{\partial\left\langle\phi_{l} \beta_{i}^{\prime} \beta_{i}^{\prime \prime}\right\rangle}{\partial t}+\lambda\left[\left(1+P_{M}\right)\left(k^{2}+k^{\prime 2}\right)+2 P_{M} k_{k} k_{k}^{\prime}\right. \\
& \left.+2 \frac{R}{\lambda}-\frac{f}{\lambda}\right]\left\langle\phi_{l} \beta_{i}^{\prime} \beta_{i}^{\prime \prime}\right\rangle \\
& =i\left(k_{k}+k_{k}^{\prime}\right)\left\langle\phi_{l} \phi_{k} \beta_{i}^{\prime} \beta_{i}^{\prime \prime}\right\rangle-i\left(k_{k}+k_{k}^{\prime}\right)\left\langle\beta_{l} \beta_{k} \beta_{i}^{\prime} \beta_{i}^{\prime \prime}\right\rangle \\
& -i\left(k_{k}+k_{k}^{\prime}\right)\left\langle\phi_{l} \phi_{k}^{\prime} \beta_{i}^{\prime} \beta_{i}^{\prime \prime}\right\rangle+i\left(k_{k}+k^{\prime}\right)\left\langle\phi_{l} \phi_{i}^{\prime} \beta_{i}^{\prime} \beta_{i}^{\prime \prime}\right\rangle \\
& +i\left(k_{l}+k_{l}^{\prime}\right)\left\langle\gamma \beta_{i}^{\prime} \beta_{i}^{\prime \prime}\right\rangle-f\left\langle\delta_{l} \beta_{i}^{\prime} \beta_{i}^{\prime \prime}\right\rangle
\end{aligned}
$$

To relate the terms on right hand side of equation (32) derived from the quadruple correlation terms and from the pressure force term in equation (22), we take the derivative with respect to $x_{j}$ of the momentum equation (17) for the point $p$, and combine with the continuity equation to give

$-\frac{\partial^{2}\langle w\rangle}{\partial x_{l} \partial x_{l}}=\frac{\partial^{2}}{\partial x_{l} \partial x_{k}}\left(u_{l} u_{k}-h_{l} h_{k}\right)$

Multiplying equation (33) by $h_{i}^{\prime} h_{j}^{\prime \prime}$ taking time averages and writing this equation in terms of the independent variables $\hat{r}$ and $\hat{r^{\prime}}$

$-\left[\frac{\partial^{2}}{\partial r_{l} \partial r_{l}}+2 \frac{\partial^{2}}{\partial r_{l} \partial r_{l}^{\prime}}+\frac{\partial^{2}}{\partial r_{l}^{\prime} \partial r_{l}^{\prime}}\right]\left\langle w h_{i}^{\prime}{ }^{\prime \prime}{ }_{j}\right\rangle$

$=\left[\frac{\partial^{2}}{\partial r_{l} \partial r_{k}}+\frac{\partial^{2}}{\partial r_{l}^{\prime} \partial r_{k}}+\frac{\partial^{2}}{\partial r_{l} \partial r_{k}^{\prime}}+\frac{\partial^{2}}{\partial r_{l}^{\prime} \partial r_{k}^{\prime}}\right]$

$\times\left(\left\langle u_{l} u_{k} h_{i}^{\prime} h_{j}^{\prime \prime}\right\rangle-\left\langle h_{l} h_{k} h_{i}^{\prime}{ }^{\prime \prime}{ }_{j}\right\rangle\right)$ 
Now taking the Fourier transforms of equation (34) we get

$-\left\langle\gamma \beta_{i}^{\prime} \beta_{j}^{\prime \prime}\right\rangle=$

$\frac{\left(k_{l} k_{k}+k_{l}^{\prime} k_{k}+k_{l} k_{k}^{\prime}+k_{l}^{\prime} k_{k}^{\prime}\right)\left(\left\langle\phi_{l} \phi_{k} \beta_{i}^{\prime} \beta_{j}^{\prime \prime}\right\rangle-\left\langle\beta_{l} \beta_{k} \beta_{i}^{\prime} \theta \beta_{j}^{\prime \prime}\right\rangle\right)}{k^{2}+2 k_{l} k_{l}^{\prime}+k^{\prime 2}}$

Thus the equations (34) and (35) are the spectral equation corresponding to the three-point correlation equations. Equation (35) can be used to eliminate $\left\langle\mathcal{\beta}_{i}^{\prime} \beta_{j}^{\prime \prime}\right\rangle$ from the equation (32).

\section{Solution for times before the final period}

It is known that equation for final period of decay is obtained by considering the two-point correlations after neglecting the 3rd order correlation terms. To study the decay for times before the final period, the three point correlations are considered and the quadruple correlation terms are neglected because the quadruple correlation terms decays faster than the lower-order correlation terms. But to get a better picture of decay of the MHD homogeneous turbulence in presence of dust particle from its initial period to its final period , three-point correlation equations are to be considered. Here, we neglect the quadruple correlation terms since the decay faster than the lower order correlation terms.

Putting the value of $\left\langle\beta_{i}^{\prime} \beta_{j}^{\prime \prime}\right\rangle$ from equation (35) into equation (32 and neglecting all the quadruple correlation terms, we have

$\frac{\partial\left\langle\phi_{l} \beta_{i}^{\prime} \beta_{i}^{\prime \prime}\right\rangle}{\partial t}+\lambda\left[\left(1+P_{M}\right)\left(k^{2}+k^{\prime 2}\right)+2 P_{M} k_{k} k_{k}^{\prime}\right.$

$\left.+2 \frac{R}{\lambda}-\frac{f}{\lambda}\right]\left\langle\phi_{l} \beta_{i}^{\prime} \beta_{i}^{\prime \prime}\right\rangle+f\left\langle\delta_{l} \beta_{i}^{\prime} \beta_{i}^{\prime \prime}\right\rangle=0$

$\Rightarrow \frac{\partial\left\langle\phi_{l} \beta_{i}^{\prime} \beta_{i}^{\prime \prime}\right\rangle}{\partial t}+\lambda\left[\left(1+P_{M}\right)\left(k^{2}+k^{\prime 2}\right)\right.$

$\left.+2 P_{M} k_{k} k_{k}^{\prime}+2 \frac{R}{\lambda}-\frac{f s}{\lambda}\right]\left\langle\phi_{l} \beta_{i}^{\prime} \beta_{i}^{\prime \prime}\right\rangle=0$

where $\left\langle\delta_{l} \beta_{i}^{\prime} \beta_{i}^{\prime \prime}\right\rangle=\mathrm{C}\left\langle\phi_{l} \beta_{i}^{\prime} \beta_{i}^{\prime \prime}\right\rangle$ and $1-\mathrm{C}=\mathrm{S}, \mathrm{C}$ and $\mathrm{S}$ are arbitrary constants.

Taking inner multiplication by $k_{l}$, we get

$\frac{\partial\left(k_{l}\left\langle\phi_{l} \beta_{i}^{\prime} \beta_{i}^{\prime \prime}\right\rangle\right)}{\partial t}+\lambda\left[\left(1+P_{M}\right)\left(k^{2}+k^{\prime 2}\right)+2 P_{M} k_{k} k_{k}^{\prime}\right.$. $\left.+\frac{2 R}{\lambda}-\frac{f s}{\lambda}\right]\left(K_{l}\left\langle\phi_{l} \beta_{i}^{\prime} \beta_{i}^{\prime \prime}\right\rangle\right)=0$

Integrating the equation (37) between t0 and $t$, and gives

$k_{l}\left\langle\phi_{l} \beta_{i}^{\prime} \beta_{i}^{\prime \prime}\right\rangle=k_{l}\left[\left\langle\phi_{l} \beta_{i}^{\prime} \beta_{i}^{\prime \prime}\right\rangle_{0}\right] \exp \left\{-\lambda\left[\left(\left(1+P_{M}\right)\left(k^{2}+k^{\prime 2}\right)\right.\right.\right.$

$\left.\left.+2 P_{M} k_{k} k^{\prime} \cos \theta+\frac{2 R}{\lambda}-\frac{f s}{\lambda}\right](\mathrm{t}-\mathrm{t} 0)\right\}$

where $\theta$ is the angle between $\hat{k}$ and $k^{\prime}$ and $\left\langle\phi_{l} \beta_{i}^{\prime} \theta_{i}^{\prime \prime}\right\rangle_{0}$ is the value of $\left\langle\phi_{l} \beta_{i}^{\prime} \theta_{i}^{\prime \prime}\right\rangle$ at $\mathrm{t}=$ to.

Now, by letting $r$ ' in equation (23) and comparing with equations (12) and (13), we get

$$
\begin{aligned}
& \left\langle\alpha_{i} \psi_{k} \psi_{i}^{\prime}(\hat{k})\right\rangle=\int_{-\infty}^{\infty}\left\langle\phi_{l} \beta_{i}^{\prime}(\hat{k}) \beta_{i}^{\prime \prime}\left(\hat{k}^{\prime}\right)\right\rangle d \hat{k}^{\prime} \\
& \left\langle\alpha_{k} \psi_{i} \psi_{i}^{\prime}(-\hat{k})\right\rangle=\int_{-\infty}^{\infty}\left\langle\phi_{l} \beta_{i}^{\prime}(-k) \beta_{i}^{\prime \prime}\left(-k^{\prime}\right)\right\rangle d \hat{k}^{\prime}
\end{aligned}
$$

Substituting equation (38) - (40) in equation (16), we get

$\frac{\partial\left\langle\psi_{i} \psi_{i}^{\prime}(\hat{k})\right\rangle}{\partial t}+2 \lambda\left[k^{2}+\frac{R}{\lambda}\right]\left\langle\psi_{i} \psi_{i}^{\prime}(\hat{k})\right\rangle=\int_{-\infty}^{\infty} 2 i k_{l}$

$\left[\left\langle\phi_{l} \beta_{i}^{\prime}(\hat{k}) \beta_{i}^{\prime \prime}\left(\hat{k}^{\prime}\right)\right\rangle-\left\langle\phi_{l} \beta_{i}^{\prime}\left(-\hat{k}^{\prime}\right) \beta_{i}^{\prime \prime}\left(-\hat{k}^{\prime}\right)\right\rangle\right]$

$\times \exp \left[-\lambda\left\{\left(1+p_{M}\right)\left(k^{2}+k^{\prime 2}\right)+2 P_{M} k^{\prime} \cos \theta+\right.\right.$

$\left.\left.+\frac{2 R}{\lambda}-\frac{f s}{\lambda}\right\}\left(t-t_{0}\right)\right] d \hat{K}^{\prime}$

Now, $d \hat{k}^{\prime}$ can be expressed in terms of $k^{\prime}$ and $\theta$ that is $d \hat{K}^{\prime}=-2 \pi k^{\prime 2} d(\cos \theta) d k^{\prime}$ (cf. Deissler,1960).

With the above relation, equation (41) to give

$$
\frac{\partial\left\langle\psi_{i} \psi_{i}{ }^{\prime}(\hat{k})\right\rangle}{\partial t}+2 \lambda\left[k^{2}+\frac{R}{\lambda}\right]\left\langle\psi_{i} \psi_{i}^{\prime}(\hat{k})\right\rangle=2 \int_{-\infty}^{\infty} 2 i k_{l}
$$

$\left[\left\langle\phi_{l} \beta_{i}^{\prime}(\hat{k}) \beta_{i}^{\prime \prime}\left(\hat{k}^{\prime}\right)\right\rangle-\left\langle\phi_{l} \beta_{i}^{\prime}\left(-\hat{k}^{\prime}\right) \beta_{i}^{\prime \prime}\left(-\hat{k}^{\prime}\right)\right\rangle\right]_{0}$

$\times k^{\prime 2}\left[\int_{-1}^{1} \exp \left\{-\lambda\left(t-t_{0}\right)\left[\left(1+P_{M}\right)\left(k^{2}+k^{\prime 2}\right)+2 P_{M} k k^{\prime}\right.\right.\right.$, 
$\left.\left.\left.\cos \theta+\frac{2 R}{\lambda}-\frac{f S}{\lambda}\right]\right\} d(\cos \theta)\right] d \hat{K}^{\prime}$

In order to find the solution completely and following (Loeffler and Deissler,1961) we assume that

$$
\begin{aligned}
& i k_{l}\left[\left\langle\phi_{l} \beta_{i}^{\prime}(\hat{K}) \beta_{i}^{\prime \prime}\left(\hat{K}^{\prime}\right)\right\rangle-\left\langle\phi_{l} \beta_{i}^{\prime}(-\hat{k}) \beta_{i}^{\prime \prime}\left(-\hat{k}^{\prime}\right)\right\rangle\right]_{0} \\
& =-\frac{\xi_{0}}{(2 \pi)^{2}}\left[k^{2} k^{\prime 4}-k^{4} k^{\prime 2}\right]
\end{aligned}
$$

where $\xi_{0}$ is a constant depending on the initial conditions. The negative sign is placed in front of $\xi_{0}$ in order to make the transfer of energy from small large wave numbers for positive value of $\xi_{0}$.

Substituting equation (43) into equation (42) and completing the integration with respect to $\cos \theta$ one obtains

$$
\frac{\partial\left(2 \pi\left\langle\psi_{i} \psi_{i}^{\prime}(\hat{K})\right\rangle\right.}{\partial t}+2 \lambda\left[k^{2}+\frac{R}{\lambda}\right]\left(2 \pi\left\langle\psi_{i} \psi_{I}^{\prime}(\hat{K})\right\rangle\right)=-\frac{\xi_{0}}{v\left(t-t_{O}\right)}
$$$$
\int_{0}^{\infty}\left(k^{3} k^{5}-k^{5} k^{3}\right) \times\left[\operatorname { e x p } \left\{-\lambda\left(t-t_{o}\right)\left[\left(1+P_{M}\right)\left(k^{2}+k^{\prime 2}\right)\right.\right.\right.
$$$$
\left.\left.-2 P_{M} k k^{\prime}+\frac{2 R}{\lambda}-\frac{f S}{\lambda}\right]\right\}-\exp \left\{-\lambda\left(t-t_{o}\right)\left[\left(1+P_{M}\right)\right.\right.
$$

$\left.\left.\left.\left(k^{2}+k^{\prime 2}\right)+2 P_{M} k k^{\prime}+\frac{2 R}{\lambda}-\frac{f S}{\lambda}\right]\right\}\right] d K^{\prime}$

Multiplying both sides of equation (44) by $k^{2}$, we get

$$
\frac{\partial H}{\partial t}+2 \lambda\left(k^{2}+\frac{R}{\lambda}\right) H=G
$$

where, $\mathrm{H}=2 \pi k^{2}\left\langle\psi_{i} \psi_{k}^{\prime}(\hat{K})\right\rangle$ is the magnetic energy spectrum function and $\mathrm{G}$ is the magnetic energy transfer term is given by

$$
\begin{aligned}
& G=-\frac{\xi_{0}}{v\left(t-t_{0}\right)} \int_{0}^{\infty}\left(k^{3} k^{\prime 5}-k^{5} k^{\prime 3}\right)\left[\operatorname { e x p } \left\{-\lambda\left(t-t_{0}\right)\left[\left(1+P_{M},\right.\right.\right.\right. \\
& \left.\left.\mid\left(k^{2}+k^{\prime 2}\right)-2 P_{M} k k^{\prime}+\frac{2 R}{\lambda}-\frac{f S}{\lambda}\right]\right\}-\exp \left\{-\lambda\left(t-t_{0}\right) \mid\right. \\
& \left.\left.\left[\left(1+P_{M}\right)\left(k^{2}+k^{\prime 2}\right)+2 P_{M} k k^{\prime}+\frac{2 R}{\lambda}-\frac{f S}{\lambda}\right]\right\}\right] d K^{\prime}
\end{aligned}
$$

Integrating equation (46) with respect to $K$ ', we have

$$
G=\frac{-\xi_{0} P_{M} \sqrt{\pi}}{4 \lambda^{3 / 2}\left(t-t_{0}\right)^{3 / 2}\left(1+P_{M}\right)^{5 / 2}} \exp \left[-\left\{2 R-\frac{f S}{\lambda}\right\}\right.
$$$$
\left.\left(t-t_{0}\right)\right] \exp \left[-\lambda\left(t-t_{0}\right)\left(\frac{1+2 P_{M}}{1+P_{M}}\right) k^{2}\right]
$$$$
\times\left[\frac{15 P_{M} k^{4}}{4 v^{2}\left(t-t_{0}\right)^{2}\left(1+P_{M}\right)}+\frac{1}{\left(t-t_{0}\right)}\left\{\frac{5 P_{M}^{2}}{v\left(1+P_{M}\right)^{2}}-\frac{3}{2 v}\right\}\right.
$$

$$
\left.\cdot k^{6}+\frac{P_{M}}{\left(1+P_{M}\right)}\left\{\frac{P_{M}^{2}}{\left(1+P_{M}\right)^{2}}-1\right\} k^{8}\right]
$$

The series of equation (42) contains only even powers of $k^{4}$ and start with and the equation represents the transfer function arising owing to consideration of magnetic field at three points at a time.

It is interesting to note that if we integrate equation (47) over all wave numbers, we find that

$$
\int_{0}^{\infty} G d k=0
$$

which indicates that the expression for $\mathrm{G}$ satisfies the condition of continuity and homogeneity.

The linear equation (45) can be solved to give

$$
H=\exp \left[-2 \lambda\left(k^{2}+\frac{R}{\lambda}\right)\left(t-t_{o}\right)\right] \int G \exp \left[2 \lambda\left(k^{2}+\frac{R}{\lambda}\right)\right.
$$

$\left.\prime\left(t-t_{o}\right)\right] d t+J(k) \exp \left[-2 \lambda\left(k^{2}+\frac{R}{\lambda}\right)\left(t-t_{o}\right)\right]$

where $\mathrm{J}(\mathrm{K})=\frac{N_{0} k^{2}}{\pi}$ is a constant of integration and can be obtained as by (Corrsin,1951). Substituting the values of G from equation (47) in to equation (49) and integrating with respect to $t$, we get

$$
\begin{aligned}
& H=\frac{N_{0} k^{2}}{\pi} \exp \left[-2 \lambda\left(k^{2}+\frac{R}{\lambda}\right)\left(t-t_{o}\right)\right] \\
& +\frac{\xi_{0} \sqrt{\pi} p_{M}}{4 \lambda^{3 / 2}\left(1+P_{M}\right)^{7 / 2}} \times \exp \left[-\{2 R-f S\}\left(t-t_{o}\right)\right] \\
& \exp \left[-\lambda k^{2}\left\{\frac{1+2 p_{M}}{\left(1+p_{M}\right)}\right\}\left(t-t_{o}\right)\right] \times\left[\frac{3 p_{M}{ }^{r} k^{4}}{2 \lambda^{2} P_{M}\left(t-t_{o}\right)^{5 / 2}}\right.
\end{aligned}
$$


$+\frac{\left(7 p_{M}-6\right) k^{6}}{3 \lambda\left(1+p_{M}\right)\left(t-t_{o}\right)^{3 / 2}}-\frac{4\left(3 p_{M}^{2}-2 p_{M}+3\right) k^{8}}{3\left(1+p_{M}\right)^{2}\left(t-t_{o}\right)^{1 / 2}}$

$\left.+\frac{8 \lambda^{1 / 2}\left(3 p_{M}^{2}-2 p_{M}+3\right) k^{9}}{3\left(1+p_{M}\right)^{5 / 2}} N(\omega)\right]$

Where $N(\omega)=e^{-\omega^{2}} \int_{o}^{\omega} e^{x^{2}} d x, \omega=k \sqrt{\frac{\lambda\left(t-t_{o}\right)}{\left(1+p_{M}\right)}}$.

By setting $\hat{r}=0, j=i, d \hat{K}=-2 \pi k^{2} d(\cos \theta) d \hat{k}$ and $H=2 \pi k^{2}\left\langle\psi_{i} \psi_{j}^{\prime}(\hat{K})\right\rangle$ in equation (9), we get the expression for magnetic energy decay with the fluctuating concentration as

$\frac{\left\langle h^{2}\right\rangle}{2}=\frac{\left\langle h_{i} h_{i}^{\prime}\right\rangle}{2}=\int_{0}^{\infty} H d \hat{k}$

Substituting equation (50) in to (51) and after integration with respect to $\mathrm{k}$, we get

$\frac{\left\langle h^{2}\right\rangle}{2}=\exp \left[-2 R\left(t-t_{0}\right)\right]\left[\frac{N_{o}\left(t-t_{o}\right)^{-3 / 2}}{8 \lambda^{3 / 2} \sqrt{2 \pi}}+\right.$

$\exp [f S\}] \times \frac{\xi_{0} \pi\left(t-t_{o}\right)^{-5}}{4 \lambda^{6}\left(1+p_{M}\right)\left(1+2 p_{M}\right)}$

$\times\left\{\frac{9}{16}+\frac{5 p_{M}\left(7 p_{M}-6\right)}{16\left(1+2 p_{M}\right)}-\frac{35 p_{M}\left(3 p_{M}^{2}-2 p_{M}+3\right)}{8\left(1+2 p_{M}\right)^{2}}+\right.$

$\left.\left.\frac{8 p_{M}\left(3 p_{M}^{2}-2 p_{M}+3\right)}{3.2^{6} \cdot\left(1+2 p_{M}\right)^{3}} \sum_{n=0}^{\infty} \frac{1.3 .5 \ldots .(2 n+9)}{n !(2 n+1) 2^{2 n}\left(1+p_{M}\right)^{n}}\right\}\right]$

or $\frac{\left\langle h^{2}\right\rangle}{2}=\exp \left[-2 R\left(t-t_{0}\right)\right]\left[\frac{N_{o}\left(t-t_{o}\right)^{-3 / 2}}{8 \lambda^{3 / 2} \sqrt{2 \pi}}+\right.$

$\left.\exp [f S\}] \xi_{0} Z\left(t-t_{o}\right)^{-5}\right]$

where

$$
Z=\frac{\pi}{\left(1+p_{M}\right)\left(1+2 p_{M}\right)^{5 / 2}}
$$

$$
\begin{gathered}
-\left[\frac{9}{16}+\frac{5 p_{M}\left(7 p_{M}-6\right)}{16\left(1+2 p_{M}\right)}-\frac{35 p_{M}\left(3 p_{M}^{2}-2 p_{M}+3\right)}{8\left(1+2 p_{M}\right)^{2}}\right. \\
\left.+\frac{8 p_{M}\left(3 p_{M}^{2}-2 p_{M}+3\right)}{3.2^{6} \cdot\left(1+2 p_{M}\right)^{3}}+----\right]
\end{gathered}
$$

Thus the decay law for magnetic energy fluctuation of dusty fluid MHD turbulence governing the concentration of a dilute contaminant undergoing a first order chemical reaction before the final period may be written as

$$
\begin{aligned}
& \left\langle h^{2}\right\rangle=\exp \left[-2 R\left(t-t_{0}\right)\right]\left[A\left(t-t_{0}\right)^{-3 / 2}+\right. \\
& \left.\exp [f s\}] B\left(t-t_{0}\right)^{-5}\right]
\end{aligned}
$$

where, $\quad A=\frac{N_{0}}{8 \lambda^{3 / 2} \sqrt{2 \pi}} \quad, \quad B=\xi_{0} Z$

\section{Results and Discussion}

In equation (53) we obtained the decay law for magnetic energy fluctuation of dusty MHD turbulence governing the concentration of a dilute contaminant undergoing a first order chemical reaction before the final period considering three-point correlation after neglecting quadruple correlation terms. If the the fluid is clean, i.e. $f=0$ then the equation (53) becomes

$\left\langle h^{2}\right\rangle=\exp \left[-2 R\left(t-t_{0}\right)\right]\left[A\left(t-t_{0}\right)^{-3 / 2}+B\left(t-t_{0}\right)^{-5}\right]$

which was obtained earlier by (Sarker and Islam,2001)

In absence of chemical reaction, i.e, $\mathrm{R}=0$ then the equation (54) becomes

$\left\langle h^{2}\right\rangle=\left[A\left(t-t_{0}\right)^{-3 / 2}+B\left(t-t_{0}\right)^{-5}\right]$

which was obtained earlier by (Sarker and Kishore,1991).

\section{Conclusion}

This study shows that due to the effect of dust particles in the magnetic field with chemical reaction of the first order in the concentrarion the magnetic field fluctuation i,e.the turbulent energy decays more rapidly than the energy for clean fluid and the faster rate is governed by. Here the chemical reaction $(R \neq 0)$ in dusty fluid MHD turbulence causes the concentration to decay more they would for clean fluid and it is gov- 
erned by exp $\quad\left[-\left\{2 R T_{M}-f_{s}\right\}\right] \quad$ The first term of right hand side of equation (53) corresponds to the energy of magnetic field fluctuation of concentration for the two-point correlation and the second term represents magnetic energy for the three-point correlation. In equation (53), the term associated with the three-point correlation dies out faster than the two-point correlation. For large times the last term in the equation (53) becomes negligible, leaving the $-3 / 2$ power decay law for the final period. If higher order correlations are considered in the analysis, it appears that more terms of higher power of time would be added to the equation (53).

\section{Acknowledgement}

The authors would like to thanks the reviewer for his valuable comments and suggestions. The authers also express their gratitude to the Department of Applied Mathematics, University of Rajshahi for providinng us all facilities regarding this article.

\section{References}

Azad M. A. K and Sarker M. S. A (2003) Decay of MHD turbulence before the final period for the case of multipoint and multi-time in presence of dust particle. The BJSIR 38(3-4): 151-164.

Chandrasekhar S. (1951a) The invariant theory of isotropic turbulence in magneto hydrodynamics. Proc. Roy. Soc. London, A 204, 435-449.

Corrsin S. (1951b) On the spectrum of isotropic temperature fluctuations in isotropic turbulence. J. Applied physics. 22: 469-473.

Deissler R. G (1958) On the decay of homogeneous turbulence before the final period. J. Phys.Fluid. 1: 111-121.

Deissler R. G (1960) Decay law of homogeneous turbulence for time before the final period. J. Phys. Fluid. 3: 176187.

Islam M. A and Sarker M. S. A (2001) First order reactant in MHD turbulence before the final period of decay for the case of multi-point and multi-time. Indian J. Pure and Appl. Math. 32(8): 1173-1184.
Kumar P. and Patel S. R. (1974) First order reactant in homogeneous turbulence before the final period for the case of multi-point and single time. J. Physics of Fluids. 17: 1362.

Kumar P. and Patel S. R.(1975) First order reactant in homogeneous turbulence before the final period for the case of multi-point and multi-time. Int. J. Engng. Sci. 13: 305-315.

Loefter A. L and Deissler R. G. (1961) Decay of temperature fluctuations in homogeneous turbulence before the final period. Int. J. Heat mass Transfer. 1: 312-324.

Patel S. R. (1976) First order reactant in homogeneous turbulence-Numerical results. Int. J. Engng. Sci.14: 75.

Sarker M. S. A and Kishore N. (1991) Decay of MHD turbulence before the final period. Int. J. Engng. Sci. 29: 1479-1485.

Sarker M. S. A (1993) Vorticity covariance of dusty fluid turbulence in a rotating frame. In: Proceedings of the Ninth Bangladesh Mathematical Conference, Rajshahi Univ. Cont (1993), pp 127.

Sarker M. S. A. and Islam M.A. (2001) First order reactant in MHD Turbulence before the final period of decay. Ph.D. Thesis, Dept. of Mathematics, Rajshahi University, Rajshahi. pp 56-70.

Sarker M. S. A and Islam,M.A (2001) Decay of MHD turbulence before the final period for the case of multi-point and multi-time. Indian J. Pure and Appl. Math.32(7): 1065-1076.

Sarker M. S. A and Islam M. A (2001) Decay of dusty fluid turbulence before the final period in a rotating system. J. Math and Math .Sci .16: 35-48.

Received : January 25, 2009;

Accepted : July 30, 2009 